\title{
Effect of long non-coding RNA PVT1 on cell proliferation and migration in melanoma
}

\author{
LAMEI CHEN $^{1 *}$, DONGMEI MA ${ }^{1 *}$, YUANYUAN LI ${ }^{1}$, XIAOYING $\mathrm{LI}^{2}$, \\ LEI ZHAO ${ }^{3}$, JING ZHANG ${ }^{4}$ and YALI SONG ${ }^{1}$ \\ ${ }^{1}$ Department of Dermatology; ${ }^{2}$ Laboratory of Medicine, Shandong Provincial Hospital Affiliated to Shandong University, \\ Jinan, Shandong 250021; ${ }^{3}$ Institute of Basic Medical Sciences, Shandong University; ${ }^{4}$ Department of Anatomy, \\ School of Basic Medical Sciences, Shandong University, Jinan, Shandong 250012, P.R. China
}

Received September 19, 2016; Accepted November 13, 2017

DOI: 10.3892/ijmm.2017.3335

\begin{abstract}
The present study aimed to investigate the potential role of the long non-coding RNA (lncRNA) Pvtl oncogene (non-protein coding) (PVT1) in the progression and metastasis of malignant melanoma, and to reveal its possible molecular mechanisms. The expression of lncRNA PVT1 in melanoma tissues and adjacent normal skin from patients with melanoma, and in the melanoma A-375 and sk-mel-5 cell lines, was analyzed using reverse transcription-quantitative polymerase chain reaction and western blot analyses. The effects of PVT1 expression on cell proliferation, the cell cycle, cell migration and cell invasion were analyzed using MTT assay, flow cytometry, Transwell and scratch assays, respectively. The interaction between PVT1 and enhancer of zeste homolog 2 (EZH2) in melanoma cells was analyzed using RNA immunoprecipitation (RIP) assay. The effect of PVT1 on microRNA-200c (miR-200c) expression was analyzed by chromatin immunoprecipitation (ChIP) assay. PVT1 was highly expressed in the melanoma tissues and cells. Silencing of PVT1 significantly inhibited cell proliferation, migration and invasion, and arrested the cell cycle at the G0/G1 stage. Additionally, PVT1 silencing significantly decreased the cyclin D1 expression in the melanoma cells. The expression of E-cadherin was significantly increased and the expression of $\mathrm{N}$-cadherin and vimentin was significantly decreased in the PVT1-silenced group. The RIP assay found that endogenous PVT1 was highly enriched by EZH2 RIP compared with that of the negative control. The ChIP assay revealed that the expression of miR-200c was decreased significantly in the PVT1-silenced group compared with the controls.
\end{abstract}

Correspondence to: Professor Yali Song, Department of Dermatology, Shandong Provincial Hospital Affiliated to Shandong University, 324 Jingwuweiqi Road, Huaiyin, Jinan, Shandong 250021, P.R. China

E-mail: yalisong79@126.com

*Contributed equally

Key words: melanoma, long non-coding RNA Pvt1 oncogene (non-protein coding), cell proliferation, metastasis, enhancer of zeste homolog 2
Overall, the present study demonstrated that the IncRNA PVT1 may contribute to the tumorigenesis and metastasis of melanoma through binding to EZH2 and regulating the expression of miR-200c. IncRNA PVT1 may serve as a potential target for the therapy of melanoma.

\section{Introduction}

Malignant melanoma is the most dangerous type of skin cancer that develops from the melanocyte pigment cells (1). The worldwide incidence of this disease is increasing yearly (2) and $\sim 68,480$ associated mortalities occur in the United States each year. This disease has the proclivity to metastasize, therefore, it usually progresses into an aggressive invasive disease in $\sim 20 \%$ of patients (3). Metastatic melanoma has a poor prognosis, with a median survival time of 6-9 months and a 5-year survival rate of $5-10 \%$ (4). However, the mechanisms of its invasion and metastasis have not been clearly understood. Therefore, it is necessary to investigate melanoma biology to facilitate the development of therapeutic strategies in melanoma.

Non-coding RNAs (ncRNAs) are emerging as novel regulators in the cancer paradigm (5). Recent studies show a particular focus on long ncRNAs (lncRNAs), which are $>200$ nucleotides in length without protein-coding capacity (6). lncRNAs have been demonstrated to serve an important role in tumorigenesis $(7,8)$. Particularly, several lncRNAs, including long intergenic non-protein coding RNA 673, BRAF-activated non-protein coding RNA and HOX transcript antisense RNA, have been reported to be involved in the tumorgenesis, invasion and metastasis of melanoma via complicated regulatory mechanisms $(5,9,10)$. The lncRNA Pvt1 oncogene (non-protein coding) (PVT1) is an oncogene that is located at 8q24.21, a well-known cancer risk region (11). PVT1 has been found to be overexpressed in numerous human cancer types, including ovarian, breast and non-small cell lung cancer (12-14). However, to the best of our knowledge, the possible roles and mechanisms of PVT1 in regulating cell proliferation and metastasis in melanoma has not been reported.

The present study investigated the expression of PVT1 in melanoma and investigated the potential effects of PVT1 expression on the proliferation and metastasis of melanoma cells. The study aimed to elucidate the underlying mechanism 
of melanoma and to provide novel insights into the treatment of this disease.

\section{Materials and methods}

Patients and samples. Between January 2014 and February 2016, 37 patients who were diagnosed with melanoma in the Shandong Provincial Hospital Affiliated to Shandong University (Jinan, Shandong, China) were enrolled in the present study. The melanoma diagnosis was pathologically confirmed. Tumor tissues and their adjacent (3-5 cm away) normal skin were obtained from clinically ongoing surgical specimens. All patients provided written informed consent prior to the study. All procedures in this study were approved by the Human Ethics Committee of the Shandong Provincial Hospital Affiliated to Shandong University.

Cell culture and small interfering RNA (siRNA) transfection. The human melanoma A-375 and sk-mel-5 cell lines and normal epiderma melanocytes PIG1 cell line were purchased from the American Type Culture Collection (ATCC; Manassas, VA, USA) to use in this study. The A-375 cells and PIG1 cells (control) were cultured in RPMI-1640 medium (Gibco; Thermo Fisher Scientific, Inc., Waltham, MA, USA) and the sk-mel-5 cells were cultured in Dulbecco's modified Eagle's medium (Gibco; Thermo Fisher Scientific, Inc.). The media were supplemented with 10\% fetal bovine serum (FBS) (Gibco; Thermo Fisher Scientific, Inc.), $100 \mathrm{U} / \mathrm{ml}$ penicillin and $100 \mu \mathrm{g} / \mathrm{ml}$ streptomycin (both Invitrogen; Thermo Fisher Scientific, Inc.) in an atmosphere of $5 \% \mathrm{CO}_{2}$ at $37^{\circ} \mathrm{C}$. Culture medium was changed every 2 days.

For cell transfection, a total of $100 \mathrm{nM}$ silenced vector of PVT1 (Sangon Biotech Co., Ltd., Shanghai, China) was transfected into the cells using Lipofectamine $2000^{\circledR}$ (Invitrogen; Thermo Fisher Scientific, Inc.). The target sequences for PVT1 were as follows: Sense, 5'-GCUUGGAGGCUGAGGAGU UTT-3' and antisense, 5'-AACUCCUCAGCCUCCAAG CTT-3'. siRNA vector without silenced PVT1 sequence was transfected into the cells as a control.

3-(4,5-Dimethylthiazol-2-yl)-2,5-diphenyltetrazolium bromide (MTT) assay. Cell proliferation was detected using an MTT assay as previously described (15). Briefly, after $24 \mathrm{~h}$ of cell transfection, cells were plated into 96 -well plates at a density of $5 \times 10^{3}$ cells/well. After cultivation for $24 \mathrm{~h}$, the supernatant was discarded, and $20 \mu \mathrm{l}$ MTT was added for another $4 \mathrm{~h}$ incubation in RPMI-1640 supplemented with $10 \%$ FBS. Next, $150 \mu$ l dimethyl sulfoxide was mixed with the cells for $10 \mathrm{~min}$. Absorbance of cells in each well was observed at $570 \mathrm{~nm}$ under an absorption spectrophotometer (Olympus, Tokyo, Japan) for the cell number calculation.

Clonogenic assay. A clonogenic assay was performed with a modification of a previous method (16). In brief, following siRNA transfection for $48 \mathrm{~h}$, cells were plated into the $60-\mathrm{mm}$ tissue culture dishes in triplicate at a density of 100 cells/dish. The cells were then grown in RPMI-1640 medium containing $10 \% \mathrm{FBS}$ at $37^{\circ} \mathrm{C}$ for 14 days. Following this, the cells were fixed and stained with Diff-Quick (Medion Diagnostics, Dudingen, Switzerland) according to manufacturer's protocol, followed by air drying. Finally, colonies were counted under an inverted microscope (IX83; Olympus), and the cell number of each colony was at least 30 cells.
Cell cycle assay. Subsequent to $48 \mathrm{~h}$ of siRNA transfection, the cells were seeded into 6-well plates and cultured for $48 \mathrm{~h}$ to reach $75-80 \%$ culture confluency. Thereafter the cells were collected and fixed in $70 \%$ ethanol, washed with phosphate-buffered saline (PBS), and resuspended in $200 \mu \mathrm{l}$ PBS containing $0.5 \mathrm{mg} / \mathrm{ml}$ RNase, $0.05 \%$ Triton X-100 and $10 \mu \mathrm{g} / \mathrm{ml}$ propidium iodide (Merck KGaA, Darmstadt, Germany). Following incubation for $1 \mathrm{~h}$ at $37^{\circ} \mathrm{C}$ in the dark, the cells were analyzed immediately using a flow cytometer (BD Biosciences, San Jose, CA, USA) for cell cycle analysis. The experiment was performed in triplicates.

Migration assay. For cell migration assay, a Transwell insert was used. Subsequent to $48 \mathrm{~h}$ of siRNA transfection, the cells were incubated for $24 \mathrm{~h}$ in serum-free RPMI-1640 medium containing $0.01 \%$ bovine serum albumin (BSA) (Merck $\mathrm{KGaA}$ ). The upper layer of the Transwell was enveloped with serum-free RPMI-1640 medium, then air-dried at $4^{\circ} \mathrm{C}$. After suctioning out the medium, $50 \mu \mathrm{l}$ fresh serum-free medium that contained $10 \mathrm{~g} / \mathrm{l} \mathrm{BSA}$ was added. After being cultured at $37^{\circ} \mathrm{C}$ for $30 \mathrm{~min}$, the Transwell was put into the 24 -well plates and cultured with RPMI-1640 medium mixed with $10 \%$ FBS. Cells in the Transwell insert were suspended with serum-free RPMI-1640 medium for $48 \mathrm{~h}$. The Transwell insert was then washed with PBS to remove the upper cells on the microporous membrane and fixed in ice-cold alcohol. Finally, the Transwell insert was stained with $0.1 \%$ crystal violet at room temperature for $30 \mathrm{~min}$, and decolorized with $33 \%$ acetic acid to exclude the false positive staining.

Invasion assay. For the assessment of cell invasion, a scratch assay was used. Briefly, cells in each group were cultured in 6 -well plates at a density of $5 \times 10^{5} /$ well. A wound was made across the well with a $200-\mu 1$ pipette tip. Images of the wound were captured immediately and then the cellular invasion of cells across the wound was documented using images captured under an inverted microscope (x10 magnification). The width of the gap was detected in 5 different fields and the average width was calculated.

Reverse transcription-quantitative polymerase chain reaction (RT-qPCR). Total RNA from cancer tissues and cells was extracted using TRIzol reagent (Invitrogen; Thermo Fisher Scientific, Inc.) and then treated with RNase-free DNase I (Promega, Madison, WI, USA). The concentration and purity of the isolated RNA were detected using SMA $400 \mathrm{UV}-\mathrm{VIS}$ (Merinton Instrument, Ltd., Shanghai, China). Next, $0.5 \mu \mathrm{g} / \mathrm{ml}$ purified RNA that was mixed with nuclease-free water was used for cDNA synthesis with the PrimerScript 1st Strand cDNA Synthesis kit (Invitrogen; Thermo Fisher Scientific, Inc.). The expression of the targets in the tissues or cells was measured in an Eppendorf Mastercycler (Brinkmann Instruments, Inc., Westbury, NY, USA) using the SYBR ExScript qRT-PCR kit (Takara Biotechnology Co., Ltd., Dalian, China) at a final volume of $20 \mu \mathrm{l}$ using the standard protocol, which was as follows: $95^{\circ} \mathrm{C}$ for $3 \mathrm{~min}, 40$ cycles of $95^{\circ} \mathrm{C}$ for $10 \mathrm{sec}$ and $60^{\circ} \mathrm{C}$ for $30 \mathrm{sec}$. Each reaction was performed in triplicate, and the $2^{-\Delta \Delta \mathrm{Cq}}$ method (17) was used to determine the relative gene expression levels. The melting curve of the amplification products was analyzed at the end of each PCR to confirm that 
Table I. Primers used for target amplification.

\begin{tabular}{lll}
\hline Name & \multicolumn{1}{c}{ Forward primers $\left(5^{\prime}-3^{\prime}\right)$} & \multicolumn{1}{c}{ Reverse primers $\left(5^{\prime}-3^{\prime}\right)$} \\
\hline PVT1 & TGAGAACTGTCCTTACGTGACC & AGAGCACCAAGACTGGCTCT \\
Cyclin D1 & CAAATGGAGCTGCTCCTGGTG & CTTCGATCTGCTCCTGGCAGG \\
Cyclin E1 & GCCAGCCTTGGGACAATAATG & GCCAGCCTTGGGACAATAATG \\
Cyclin A1 & CAAGGTCCTGATGCTTGTCA & CCCATGGTCAGAGAGCACTT \\
E-cadherin & TGTAGTTACGTATTTATTTTAGTGGCGTC & CGAATACGATCGAATCGAACCG \\
N-cadherin & AACTCCAGGGGACCTTTTC & CAAATGAAACCGGGCTATC \\
Vimentin & TCCAAGTTGCTGACCTCTC & TCAACGGCAAAGTTCTCTTC \\
GAPDH & TTCGACAGTCAGCCGCATCTT & ATCCGTTGACTCCGACCTTCA
\end{tabular}

PVT1, Pvt1 oncogene (non-protein coding); GAPDH, glyceraldehyde 3-phosphate dehydrogenase.

only one product was amplified and detected. Glyceraldehyde 3-phosphate dehydrogenase (GAPDH) was chosen as the internal control for mRNA or lncRNAs. Primers used for targets amplification are shown in Table I.

Western blotting. The cells were cultured for $48 \mathrm{~h}$ after transfection, then they were lysed with RIPA lysis and extraction buffer (Sangon Biotech Co., Ltd.). The supernatant was collected for the measurement of protein concentrations using a bicinchoninic acid protein assay kit (Pierce; Thermo Fisher Scientific.). Next, $20 \mu \mathrm{g}$ protein was subjected to a $10 \%$ sodium dodecyl sulfate-polyacrylamide gel electrophoresis and then transferred to polyvinylidene fluoride membrane (EMD Millipore, Billerica, MA, USA). Subsequent to being blocked with Tris-buffered saline Tween-20 (TBST) containing 5\% skimmed milk at room temperature for $1 \mathrm{~h}$, the membrane was incubated with rabbit anti-human primary antibodies against cyclin D1 (cat. no. 2978), cyclin E1 (cat. no. 20808) and cyclin A1 (cat. no. PA5-16519), E-cadherin (cat.no. 3195), N-cadherin (cat. no. 13116), vimentin (cat. no. 5741), and GAPDH (cat. no. 5174) (all 1:1,000 dilution) at $4^{\circ} \mathrm{C}$ overnight. Following this, the membrane was washed with 1X TBST 3 times and then incubated with horseradish peroxidase-labeled goat anti-rabbit secondary antibody (1:1,000 dilution; cat. no. 14708) at room temperature for $1 \mathrm{~h}$. All antibodies were purchased from Santa Cruz Biotechnology, Inc. (Dallas, TX, USA), except for cyclin A1 that was purchased from Thermo Fisher Scientific, Inc. Detection was conducted using the development of X-ray after the addition of a chromogenic substrate with an enhanced chemiluminescence method (EMD Millipore). GAPDH served as the internal control. Qualification of protein bands were determined using the Adobe Photoshop CS5 (Adobe, San Jose, CA, USA).

Chromatin immunoprecipitation (ChIP) assay. The ChIP assay was conducted with an EZ ChIP Chromatin Immunoprecipitation kit (EMD Millipore) according to the manufacturer's protocols. Cross-linked chromatin was sonicated into 200-1,000-bp fragments. The chromatin was immunoprecipitated using anti-c-Myc antibody (cat. no. 611451; 1:200 dilution; BD Biosciences). Normal mouse immunoglobulin G (IgG; cat. no. 554002; 1:200 dilution; BD Biosciences) was chosen as the negative control. RT-qPCR was conducted using SYBR-Green Mix (Roche Diagnostics, Indianapolis, IN, USA) as aforementioned.
RNA immunoprecipitation (RIP) assay. The RIP assay was performed using the Magna RIP RNA-Binding Protein Immunoprecipitation kit (EMD Millipore) according to the manufacturer's protocols. Anti-enhancer of zeste homolog 2 (EZH2) antibody and IgG (control) were used for RIP (Cell Signaling Technology, Inc., Danvers, MA, USA). Co-precipitated RNAs were detected by qRT-PCR analysis. Total RNAs (input controls) and isotype controls were detected simultaneously to demonstrate that the detected signals were the results of RNAs specifically binding to E-box.

Statistical analysis. Data are expressed as the mean \pm standard deviation and were analyzed using GraphPrism 5.0 software (GraphPad Software, Inc., La Jolla, CA, USA). Statistical analyses were performed using SPSS 19.0 statistical software (IBM Corp., Armonk, NY, USA). An independent samples t-test was used for the paired data significance calculation. Analysis of variance was used to calculate the significance for $>2$ groups, with Tukey's posthoc test used to calculate the difference among groups. $\mathrm{P}<0.05$ was considered to indicate a statistically significant difference.

\section{Results}

PVT1 is upregulated in melanoma tissue and cells. To investigate the role of PVT1 in melanoma, the expression levels of PVT1 in 37 paired melanoma tissues and adjacent normal skin were determined by RT-qPCR and normalization to GAPDH. Additionally, the expression levels of PVT1 in 3 melanoma cell lines were detected. The relative expression level of PVT1 was significantly higher in melanoma tissues and cells compared with that in the adjacent normal tissues and control cells $(\mathrm{P}<0.01)$. PVT1 exhibited the highest expression levels in the sk-mel-5 cells $(\mathrm{P}<0.01)$. These results suggested that melanoma progression is associated with the abnormal expression of PVT1 (Fig. 1).

Silenced PVT1 inhibits cell proliferation and arrests the cell cycle at the G0/G1 stage. As PVT1 exhibited the highest expression levels in sk-mel-5 cells, these cells were selected for cell transfection and the subsequent experiments. As shown in Fig. 2A, after sk-mel-5 cells were transfected with siRNA-PVT1, the expression of PVT1 decreased significantly $(\mathrm{P}<0.01)$. MTT and clonogenic assays found that silenced PVT1 could inhibit cell proliferation significantly $(\mathrm{P}<0.01$; Fig. $2 \mathrm{~B}-\mathrm{D})$. 

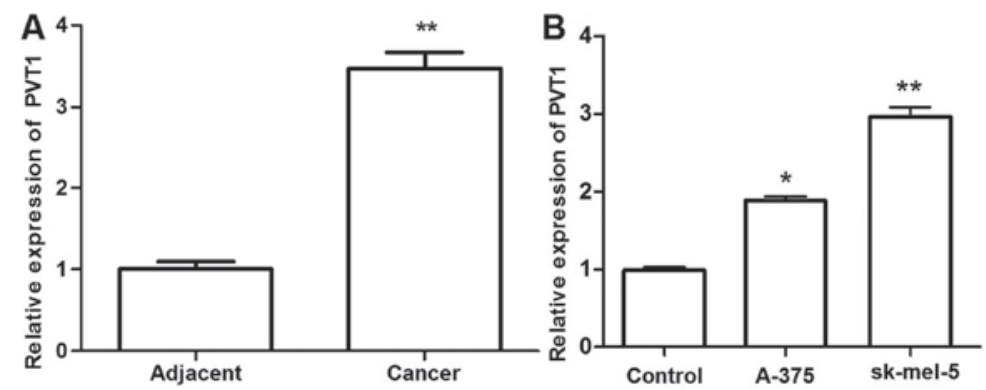

Figure 1. (A) Expression of long non-coding RNA PVT1 in melanoma tissues and (B) cell lines assayed by reverse transcription-quantitative polymerasae chain reaction. ${ }^{*} \mathrm{P}<0.05$ and ${ }^{* *} \mathrm{P}<0.01$ compared with the control/adjacent tissues. PVT1, Pvt1 oncogene (non-protein coding).

A

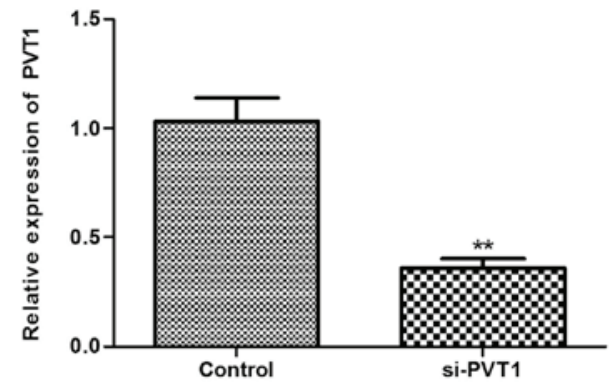

C

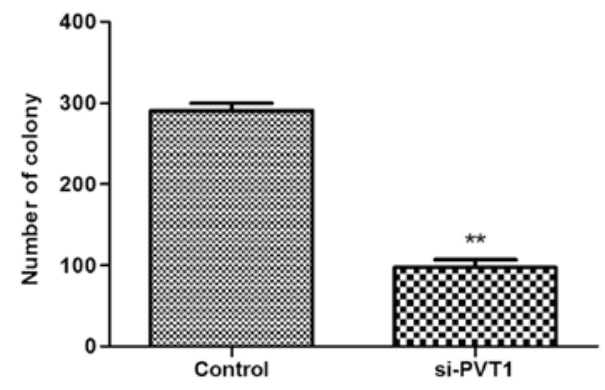

E

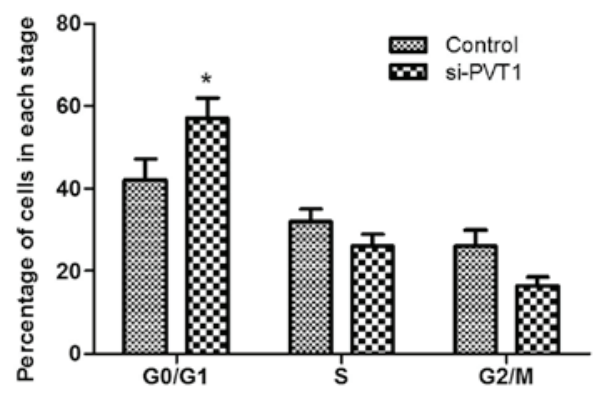

B

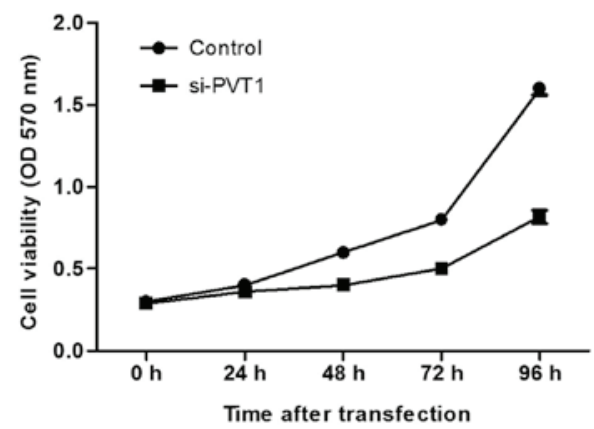

D

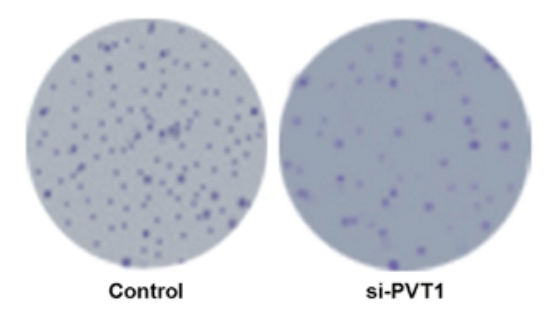

$\mathbf{F}$

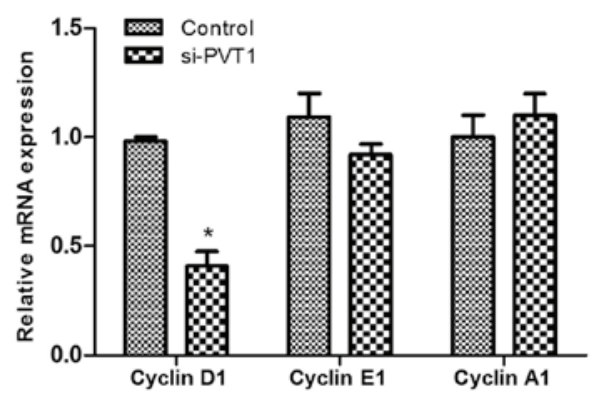

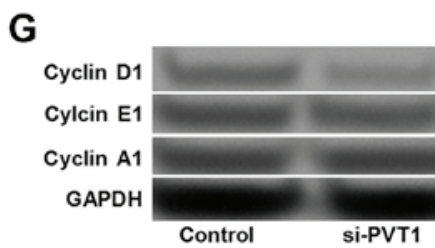

Figure 2. Effects of 1ncRNA PVT1 expression on cell proliferation and the cell cycle. (A) The expression of lncRNA PVT1 in melanoma cells following transfection with si-PVT1. (B) Silenced lncRNA PVT1 significantly inhibited melanoma cell proliferation, as assessed by MTT assay. (C and D) Silenced lncRNA PVT1 significantly decreased the number of melanoma cell colonies, as assessed by clonogenic assay. (E) Silenced lncRNA PVT1 significantly increased the percentage of cells in the G0/G1 stage. Silenced lncRNA PVT1 significantly decreased cyclin D1 expression, but had no significant effects on cyclin E1 and A1 expression in the melanoma cells, as assessed by (F) reverse transcription-quantitative polymerasae chain reaction and (G) western blot analysis. "P<0.05 and ${ }^{* *} \mathrm{P}<0.01$ compared with the control. PVT1, Pvt1 oncogene (non-protein coding); lncRNA, long non-coding RNA; si, small interfering; OD, optical density; GAPDH, glyceraldehyde 3-phosphate dehydrogenase. 


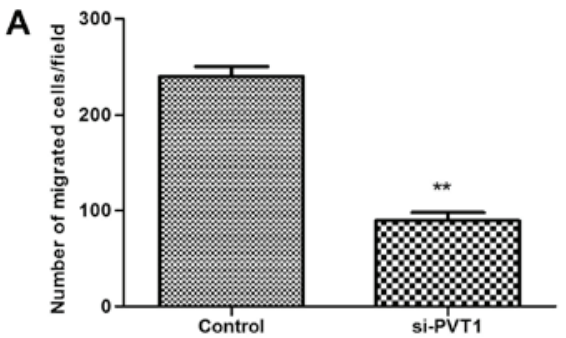

C

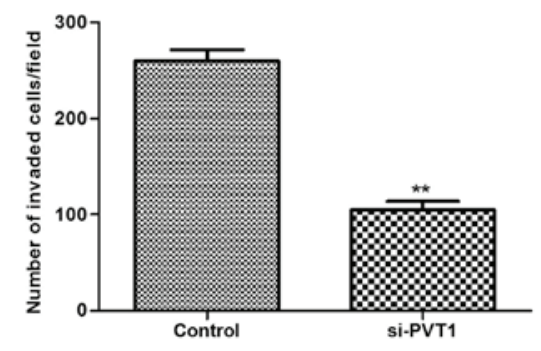

E

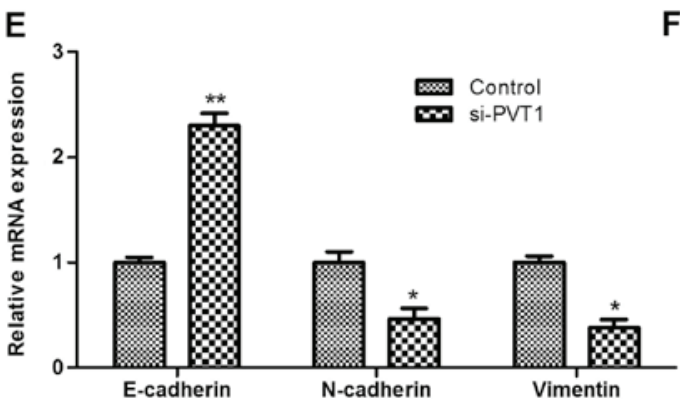

$\mathbf{F}$
B
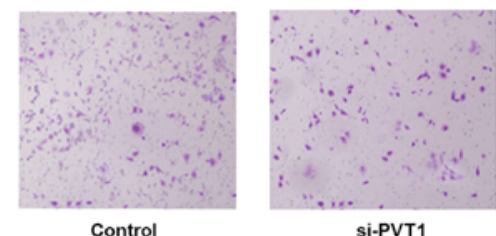

D

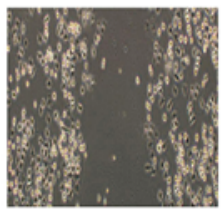

Control

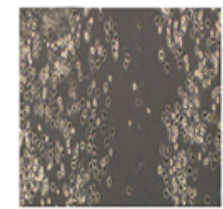

si-PVT1

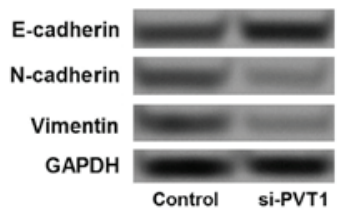

Figure 3. Effects of lncRNA PVT1 suppression on melanoma cell migration and invasion. (A and B) Transwell assays showed that silenced lncRNA PVT1 significantly decreased the number of migrated cells. (C and D) Scratch assay showed that silenced lncRNA PVT1 significantly decreased the number of invading cells. (E) Reverse transcription-quantitative polymerasae chain reaction and (F) western blot analysis showed that silenced lncRNA PVT1 significantly increased E-cadherin expression, and decreased $\mathrm{N}$-cadherin and vimentin expression. ${ }^{*} \mathrm{P}<0.05$ and ${ }^{* *} \mathrm{P}<0.01$ compared with the control. PVT1, Pvt1 oncogene (non-protein coding); lncRNA, long non-coding RNA; si, small interfering; GAPDH, glyceraldehyde 3-phosphate dehydrogenase.
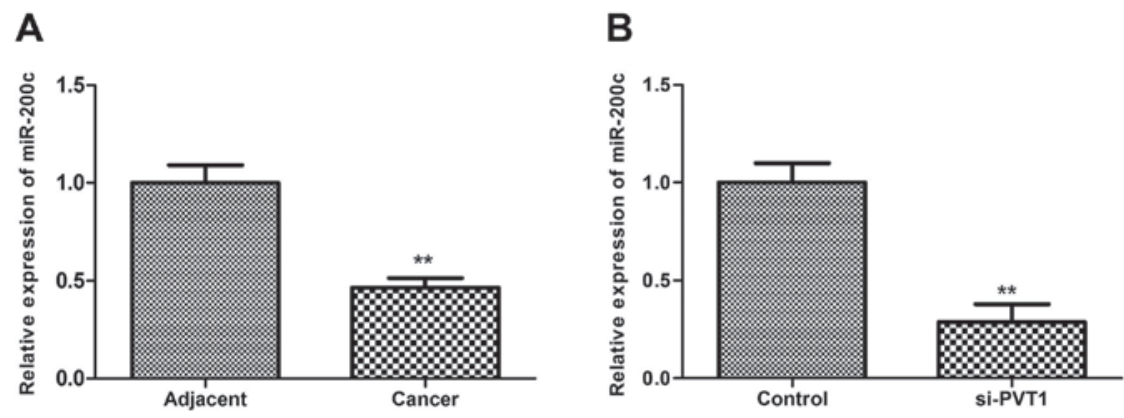

Figure 4. Effects of lncRNA PVT1 expression on miR-200c expression. (A) The expression of miR-200c in melanoma and adjacent tissues, as detected by RT-qPCR. (B) Silenced lncRNA PVT1 significantly decreased the miR-200c expression in melanoma cells detected by RT-qPCR. **P $<0.01$ compared with the control/adjacent tissue. PVT1, Pvt1 oncogene (non-protein coding); lncRNA, long non-coding RNA; si, small interfering; RT-qPCR, reverse transcriptionquantitative polymerasae chain reaction; miR-200, microRNA-200.

Additionally, the cell cycle assay revealed that silenced PVT1 significantly increased the percentage of cells at the G0/G1 stage $(\mathrm{P}<0.05)$, suggesting that the cells were arrested in the G0/G1 stage (Fig. 2E). To further verify these findings, the expression levels of cell cycle-related proteins (cyclin D1, E1 and A1) were detected. The results showed that the relative expression level of cyclin D1 decreased significantly in siRNA-PVT1 group compared with that in the control group
$(\mathrm{P}<0.05)$, while the expression of cyclin $\mathrm{A} 1$ and $\mathrm{E} 1 \mathrm{did}$ not change significantly between the two groups (Fig. 2F and G).

Silenced PVT1 suppresses cell migration and invasion by inhibiting epithelial-mesenchymal transition (EMT). To investigate the role of PVT1 in melanoma cell migration and invasion, Transwell and scratch assays were performed. The results showed that silenced PVT1 significantly inhibited the 
A
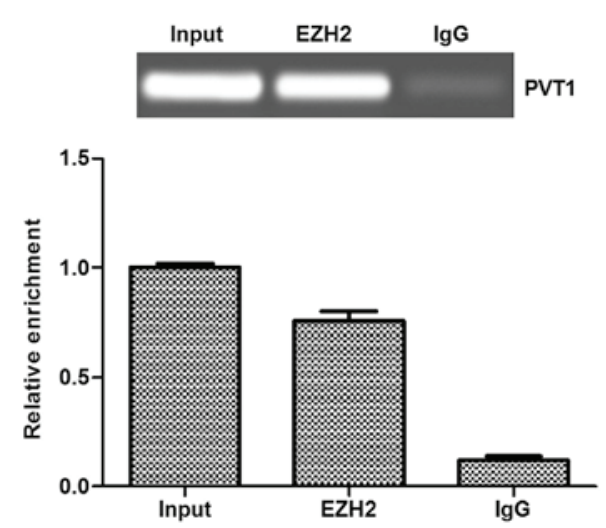
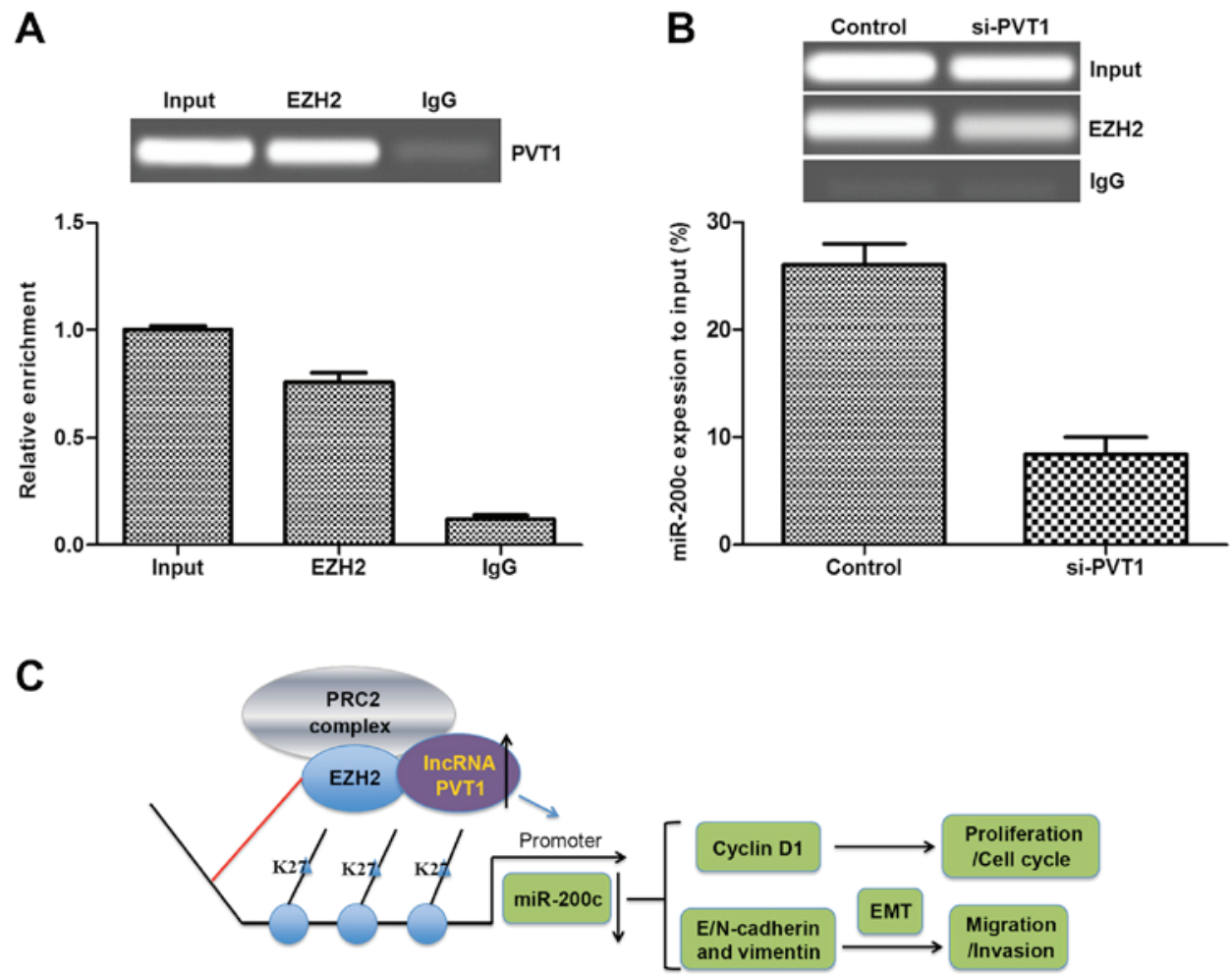

Figure 5. Effects of 1ncRNA PVT1 expression on cell metastasis were mediated by EZH2 and the regulation of miR-200c. (A) RNA immunoprecipitation assay showed that lncRNA PVT1 was significantly enriched in the EZH2 group compared with that in the IgG group. (B) Chromatin immunoprecipitation assay showed that miR-200c expression was significantly decreased in the lncRNA PVT1-silenced group compared with that in the control. (C) The regulatory mechanism of 1ncRNA PVT1 in melanoma. PVT1, Pvt1 oncogene (non-protein coding); lncRNA, long non-coding RNA; si, small interfering; EZH2, enhancer of zeste homolog 2; miR-200, microRNA-200; IgG, immunoglobulin G; EMT, epithelial-mesenchymal transition ; PRC2, polycomb repressive complex-2.

migration and invasion of sk-mel-5 cells compared with that in the control $(\mathrm{P}<0.01$; Fig. 3A-D). It has been reported that EMT plays an important role in the progression of primary tumors toward metastasis (18). Therefore, the expression levels of EMT associated biomarkers, including E-cadherin, N-cadherin and vimentin, were investigated in the present study. As shown in Fig. 3E and F, the expression of E-cadherin increased significantly in the PVT1-silenced group compared with that in the control group $(\mathrm{P}<0.01)$. By contrast, the expressions of $\mathrm{N}$-cadherin and vimentin decreased significantly in the PVT1-silenced group $(\mathrm{P}<0.05)$. These results suggested that silenced PVT1 may inhibit cell migration and invasion by inhibiting EMT.

Effects of PVT1 suppression on miR-200c expression. MicroRNA (miRNA/miR)-200 family members have been found to play a key role in tumor development (19). miR-200c, a member of the miR-200 family, has been reported to be downregulated in melanoma (20). The present study investigated the association between miR-200c and PVT1 in melanoma. In accordance with the aforementioned previous study, the present study also found that miR-200c was downregulated in melanoma tissue compared with the adjacent tissue $(\mathrm{P}<0.01$; Fig. 4A). Additionally, the results showed that miR-200c expression decreased significantly when sk-mel-5 cells were transfected with siRNA-PVT1 $(\mathrm{P}<0.01)$, which suggested that silenced PVT1 may reduce the miR-200c expression (Fig. 4B).

PVT1 expression is associated with EZH2 and regulates cell metastasis. A previous study found that EZH2 often functions as an inhibitor in cancer suppressor genes, and that lncRNAs could modulate tumor cells growth and metastasis via binding to EZH2 (21). Therefore, the present study analyzed the interactions between PVT1 and EZH2 using an RIP assay in melanoma cells. As shown in Fig. 5A, endogenous PVT1 was highly enriched by EZH2 RIP compared with the negative control (IgG RIP), implying that PVT1 may bind to EZH2. Additionally, a ChIP assay was performed to determine if silenced PVT1 affected the expression of miR-200c. As shown in Fig. 5B, the expression of miR-200c decreased significantly in the PVT1-silenced group compared with that in the control in the sk-mel-5 cells, which suggested that silenced PVT1 may alter the expression of miR-200c by targeting the promoter of miR-200c (Fig. 5C). Taken together, these results suggested that PVT1 silencing may inhibit the expression of miR-200c to inhibit the metastasis of melanoma via binding to EZH2.

\section{Discussion}

The present study examined the expression of PVT1 in melanoma tissues and cell lines. The results showed that PVT1 was overexpressed in melanoma tissues and cell lines compared with that in controls. Silenced PVT1 significantly inhibited cell proliferation, migration and invasion, and arrested the cell cycle at the G0/G1 stage. Furthermore, silenced PVT1 affected the expression of cell cycle-related proteins and EMT-associated biomarkers. Silenced PVT1 also significantly reduced the expression of miR-200c by binding to EZH2. 
An increasing number of studies have determined a role for lncRNAs in cancer $(7,22)$. In particular, an increasing amount of evidence has shown that PVT1 plays an important role in the progression of cancer, since it resides in the well-known cancer risk region $(11,23)$. For instance, Takahashi et al (24) reported that overexpression of PVT1 is involved in the prognosis of colorectal cancer by regulating cell apoptosis. Wang et al (25) found that upregulated PVT1 promotes stem cell proliferation in hepatocellular carcinoma. In accordance with these findings, the present study found that PVT1 was overexpressed in melanoma tissues and cell lines compared with that in controls, which suggested the potential role of PVT1 in melanoma.

To demonstrate the role of PVT1 in melanoma, siRNA-mediated gene silencing was used to suppress the expression of PVT1 in melanoma cells, and then the effect of silenced PVT1 on cell proliferation was investigated. The results showed that silenced PVT1 significantly inhibited cell proliferation, which was in agreement with other studies. Kong et al (26) found that PVT1-knockdown significantly inhibits gastric cancer cell proliferation in vitro and in vivo. Yang et al (14) also found that knockdown of PVT1 inhibited cell proliferation in non-small cell lung cancer. As cell proliferation is closely associated with the cell cycle, accordingly, the effects of PVT1 expression on the melanoma cell cycle and cell cycle-associated protein expression were analyzed. It was found that the silencing of PVT1 arrested the melanoma cell cycle at the G0/G1 stage, and that the expression of cyclin D1 decreased significantly. Cyclin D1 serves a vital role in promoting cancer cell proliferation (27), and has been considered as a pivotal element of malignant transformation in human cancer (28). A previous study also found that cyclin D1 was downregulated by silenced PVT1 in thyroid cancer (29). Taken together, these results indicated that downregulated cyclin D1 may be associated with the suppression of melanoma cell proliferation via the arrest of the cell cycle at the G0/G1 stage.

As aforementioned, melanoma has a proclivity to metastasize and invade (3). EMT is an biological process that gives rise to the dissemination of single carcinoma cells from the sites of the primary tumors, contributing to the progression of primary tumors toward metastasis (18). Various carcinoma cell lines undergo partial or complete EMT (30). Therefore, the present study investigated the effect of PVT1 expression on melanoma cell migration and invasion, and the expression of EMT-associated biomarkers. The data showed that silenced PVT1 significantly inhibited melanoma cell migration and invasion, increased the expression of E-cadherin and decreased the expression of $\mathrm{N}$-cadherin and vimentin significantly. During melanoma development, loss of functional E-cadherin accompanies the gain of expression of $\mathrm{N}$-cadherin (31). E-cadherin is a tumor/invasion suppressor that has been found to be downregulated in various human carcinomas, including melanoma (32). Disruption of E-cadherin-mediated homotypic cell adhesion facilitates tumor invasion (33). Additionally, one previous study also found that overexpression of vimentin in primary melanoma tissues may cause a high risk of hematogenous metastasis (34). We speculate that silenced PVT1 may inhibit melanoma cell migration and invasion by regulating EMT.

miR-200c belongs to the miR-200 family, which has been found to be involved in cancer progression (35). Particularly, the miR-200 family has been shown to regulate EMT and cell migration in cancer cell lines (36). Importantly, miR-200c has been reported to be downregulated in melanoma (20). In the present study, it was found that silenced PVT1 reduced the expression of miR-200c, indicating that silencing PVT1 may suppress the development of melanoma by downregulating miR-200c expression.

Recently, the histone methyltransferase EZH2 has attracted considerable attention due to its dysregulation in human cancer (37). Notably, PVT1 was reported to promote cell proliferation in gastric cancer by the recruitment of EZH2 (26). Thus, the interaction between EZH2 and PVT1 in melanoma was investigated in the present study and PVT1 was found to be enriched by EZH2, indicating the interaction between PVT1 and EZH2 in melanoma. Moreover, Ning et al (38) found that EZH2-mediated methylation inhibits the expression of miR-200 and promotes tumor progression. Therefore, a ChIP assay was performed in the present study to investigate whether silenced PVT1 affected the expression of miR-200c, and the resultant data found that the expression of miR-200c was decreased significantly by the silencing of PVT1 in EZH2-treated melanoma cell lines, suggesting that PVT1 suppression may decrease miR-200c level and that PVT1 may be required for EZH2 binding to the miR-200c promoter. Taken together, these results led to the hypothesis that silenced PVT1 may inhibit miR-200c expression via binding to EZH2.

In conclusion, the present study indicates that PVT1 overexpression plays an acceleratory role in the development of melanoma. Downregulation of PVT1 may inhibit melanoma cell proliferation by regulating the cell cycle, and may inhibit cell migration and invasion through the regulation of EMT, which may be achieved by inhibiting the expression of miR-200c via the binding of PVT1 to EZH2. Therefore, IncRNA PVT1 may serve as an important target in the treatment of melanoma.

\section{Acknowledgements}

The authors would like to thank Professor Xiulian Xu and Dr Wai Zhang (Chinese Academy of Medical Sciences and Peking Union Medical College, Beijing, China) for supporting this study. The authors would also like to thank Dr Jiang Hong (Key Laboratory of Cardiovascular Remodeling and Function Research, Shandong, China) for providing technical assistance.

\section{References}

1. Gray-Schopfer V, Wellbrock C and Marais R: Melanoma biology and new targeted therapy. Nature 445: 851-857, 2007.

2. Siegel RL, Miller KD and Jemal A: Cancer statistics, 2016. CA Cancer J Clin 66: 7-30, 2016.

3. Arozarena I, Sanchez-Laorden B, Packer L, Hidalgo-Carcedo C, Hayward R, Viros A, Sahai E and Marais R: Oncogenic BRAF induces melanoma cell invasion by downregulating the cGMP-specific phosphodiesterase PDE5A. Cancer Cell 19: 45-57, 2011

4. Cummins DL, Cummins JM, Pantle H, Silverman MA, Leonard AL and Chanmugam A: Cutaneous malignant melanoma. Mayo Clin Proc 81: 500-507, 2006.

5. Tang L, Zhang W, Su B and Yu B: Long noncoding RNA HOTAIR is associated with motility, invasion, and metastatic potential of metastatic melanoma. BioMed Res Int 2013: 251098-251098, 2013.

6. Muers M: RNA: genome-wide views of long non-coding RNAs. Nat Rev Genet 12: 742-743, 2011.

7. Shore AN, Herschkowitz JI and Rosen JM: Noncoding RNAs involved in mammary gland development and tumorigenesis: there's a long way to go. J Mammary Gland Biol Neoplasia 17: 43-58, 2012. 
8. Huarte M, Guttman M, Feldser D, Garber M, Koziol MJ, Kenzelmann-Broz D, Khalil AM,Zuk O, Amit I, Rabani M, et al: A large intergenic noncoding RNA induced by $\mathrm{p} 53$ mediates global gene repression in the p53 response. Cell 142: 409-419, 2010.

9. Schmidt K, Joyce CE, Buquicchio F, Brown A, Ritz J, Distel RJ, Yoon $\mathrm{CH}$ and Novina CD: The lncRNA SLNCR1 mediates melanoma invasion through a conserved SRA1-like region. Cell Reports 15: 2025-2037, 2016.

10. Flockhart RJ, Webster DE, Qu K, Mascarenhas N, Kovalski J, Kretz $\mathrm{M}$ and Khavari PA: BRAF ${ }^{\mathrm{V} 600 \mathrm{E}}$ remodels the melanocyte transcriptome and induces BANCR to regulate melanoma cell migration. Genome Res 22: 1006-1014, 2012.

11. Colombo T, Farina L, Macino G and Paci P: PVT1: a rising star among oncogenic long noncoding RNAs. BioMed Res Int 2015: 304208, 2015.

12. Guan Y, Kuo WL, Stilwell JL, Takano H, Lapuk AV, Fridlyand J, Mao JH, Yu M, Miller MA, Santos JL, et al: Amplification of PVT1 contributes to the pathophysiology of ovarian and breast cancer. Clin Cancer Res 13: 5745-5755, 2007.

13. Barsotti AM, Beckerman R, Laptenko O, Huppi K, Caplen NJ and Prives C: p53-dependent induction of PVT1 and miR-1204. J Biol Chem 287: 2509-2519, 2012.

14. Yang YR, Zang SZ, Zhong CL, Li YX, Zhao SS and Feng XJ: Increased expression of the lncRNA PVT1 promotes tumorigenesis in non-small cell lung cancer. Int J Clin Exp Pathol 7: 6929-6935, 2014.

15. Zhou Q, Chen J, Feng J and Wang J: Long noncoding RNA PVT1 modulates thyroid cancer cell proliferation by recruiting EZH2 and regulating thyroid-stimulating hormone receptor (TSHR) Tumour Biol 37: 3105-3113, 2016.

16. Franken NAP, Rodermond HM, Stap J, Haveman J and van Bree C: Clonogenic assay of cells in vitro. Nat Protoc 1: 2315-2319, 2006

17. Livak KJ and Schmittgen TD. Analysis of relative gene expression data using real-time quantitative PCR and the $2^{-\Delta \Delta C T}$ method. Methods 25: 402-408, 2001.

18. Biddle A and Mackenzie IC: Cancer stem cells and EMT in carcinoma. Cancer Metastasis Rev 31: 285-293, 2012.

19. Park SM, Gaur AB, Lengyel E and Peter ME: The miR-200 family determines the epithelial phenotype of cancer cells by targeting the E-cadherin repressors ZEB1 and ZEB2. Genes Dev 22: 894-907, 2008.

20. Xu Y, Brenn T, Brown ER, Doherty V and Melton DW: Differential expression of microRNAs during melanoma progression: miR-200c, miR-205 and miR-211 are downregulated in melanoma and act as tumour suppressors. Br J Cancer 106: 553-561, 2012.

21. Chase A and Cross NC: Aberrations of EZH2 in cancer. Clin Cancer Res 17: 2613-2618, 2011.

22. Chen $\mathrm{H}, \mathrm{Xu} J$ J, Hong J, Tang $\mathrm{R}$, Zhang $\mathrm{X}$ and Fang JY: Long noncoding RNA profiles identify five distinct molecular subtypes of colorectal cancer with clinical relevance. Mol Oncol 8: 1393-1403, 2014

23. Huang C, Yu W, Wang Q, Cui H, Wang Y, Zhang L, Han F and Huang T: Increased expression of the lncRNA PVT1 is associated with poor prognosis in pancreatic cancer patients. Minerva Med 106: 143-149, 2015.

24. Takahashi Y, Sawada G, Kurashige J, Uchi R, Matsumura T, Ueo H, Takano Y, Eguchi H, Sudo T, Sugimachi K, et al: Amplification of PVT-1 is involved in poor prognosis via apoptosis inhibition in colorectal cancers. Br J Cancer 110: 164-171, 2014.
25. Wang F, Yuan JH, Wang SB, Yang F, Yuan SX, Ye C, Yang N, Zhou WP, Li WL, Li W, et al: Oncofetal long noncoding RNA PVT1 promotes proliferation and stem cell-like property of hepatocellular carcinoma cells by stabilizing NOP2. Hepatology 60: 1278-1290, 2014

26. Kong R, Zhang EB, Yin DD, You LH, Xu TP, Chen WM, Xia R, Wan L, Sun M, Wang ZX, et al: Long noncoding RNA PVT1 indicates a poor prognosis of gastric cancer and promotes cell proliferation through epigenetically regulating p15 and p16. Mol Cancer 14: 82, 2015.

27. Gautschi O, Ratschiller D, Gugger M, Betticher DC and Heighway J: Cyclin D1 in non-small cell lung cancer: a key driver of malignant transformation. Lung Cancer 55: 1-14, 2007.

28. Li Z, Tian T, Lv F, Chang Y, Wang X, Zhang L, Li X, Li L, Ma W, Wu J, et al: Six1 promotes proliferation of pancreatic cancer cells via upregulation of cyclin D1 expression. PLoS One 8: e59203, 2013.

29. Khoo ML, Beasley NJ, Ezzat S, Freeman JL and Asa SL: Overexpression of cyclin D1 and underexpression of p27 predict lymph node metastases in papillary thyroid carcinoma. J Clin Endocrinol Metab 87: 1814-1818, 2002.

30. Thiery JP: Epithelial-mesenchymal transitions in tumour progression. Nat Rev Cancer 2: 442-454, 2002.

31. Li G, Satyamoorthy K and Herlyn M: N-cadherin-mediated intercellular interactions promote survival and migration of melanoma cells. Cancer Res 61: 3819-3825, 2001.

32. Hsu MY, Meier FE, Nesbit M, Hsu JY, Van Belle P, Elder DE and Herlyn M: E-cadherin expression in melanoma cells restores keratinocyte-mediated growth control and down-regulates expression of invasion-related adhesion receptors. Am J Pathol 156: 1515-1525, 2000.

33. Slagle BL, Zhou YZ, Birchmeier W and Scorsone KA: Deletion of the E-cadherin gene in hepatitis B virus-positive Chinese hepatocellular carcinomas. Hepatology 18: 757-762, 1993.

34. Li M, Zhang B, Sun B, Wang X, Ban X, Sun T, Liu Z and Zhao X: A novel function for vimentin: the potential biomarker for predicting melanoma hematogenous metastasis. J Exp Clin Cancer Res 29: 109, 2010

35. du Rieu MC, Torrisani J, Selves J, Al Saati T, Souque A, Dufresne M, Tsongalis GJ, Suriawinata AA, Carrère N, Buscail L, et al: MicroRNA-21 is induced early in pancreatic ductal adenocarcinoma precursor lesions. Clin Chem 56: 603-612, 2010

36. Park SM, Gaur AB, Lengyel E and Peter ME: The miR-200 family determines the epithelial phenotype of cancer cells by targeting the E-cadherin repressors ZEB1 and ZEB2. Genes Dev 22: 894-907, 2008

37. Tao T, Liu D, Liu C, Xu B, Chen S, Yin Y, Ang L, Huang Y, Zhang $\mathrm{X}$ and Chen $\mathrm{M}$ : Autoregulatory feedback loop of $\mathrm{EZH} 2 / \mathrm{miR}-200 \mathrm{c} / \mathrm{E} 2 \mathrm{~F} 3$ as a driving force for prostate cancer development. Biochim Biophys Acta 1839: 858-865, 2014.

38. Ning X, Shi Z, Liu X, Zhang A, Han L, Jiang K, Kang C and Zhang Q: DNMT1 and EZH2 mediated methylation silences the microRNA-200b/a/429 gene and promotes tumor progression. Cancer Lett 359: 198-205, 2015.

This work is licensed under a Creative Commons Attribution-NonCommercial-NoDerivatives 4.0 International (CC BY-NC-ND 4.0) License. 